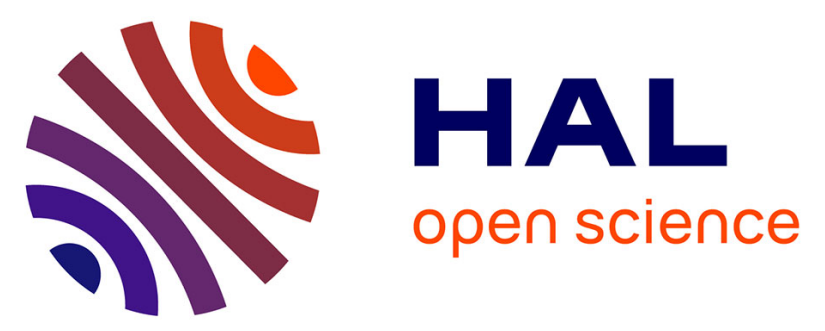

\title{
Finite Element Magnetic Models via a Coupling of Subproblems of Lower Dimensions
}

\author{
Patrick Dular, Ruth V. Sabariego, Christophe Geuzaine, Mauricio Valencia \\ Ferreira da Luz, Patrick Kuo-Peng, Laurent Krähenbühl
}

\section{- To cite this version:}

Patrick Dular, Ruth V. Sabariego, Christophe Geuzaine, Mauricio Valencia Ferreira da Luz, Patrick Kuo-Peng, et al.. Finite Element Magnetic Models via a Coupling of Subproblems of Lower Dimensions. IEEE Transactions on Magnetics, 2010, 46 (8), pp.2827 - 2830. 10.1109/TMAG.2010.2044028 . hal-00455462

\section{HAL Id: hal-00455462 https://hal.science/hal-00455462}

Submitted on 27 Jul 2010

HAL is a multi-disciplinary open access archive for the deposit and dissemination of scientific research documents, whether they are published or not. The documents may come from teaching and research institutions in France or abroad, or from public or private research centers.
L'archive ouverte pluridisciplinaire HAL, est destinée au dépôt et à la diffusion de documents scientifiques de niveau recherche, publiés ou non, émanant des établissements d'enseignement et de recherche français ou étrangers, des laboratoires publics ou privés. 


\title{
Finite Element Magnetic Models via a Coupling of Subproblems of Lower Dimensions
}

\author{
Patrick Dular ${ }^{1,2}$, Ruth V. Sabariego ${ }^{1}$, Christophe Geuzaine ${ }^{1}$, Mauricio Valencia Ferreira da Luz ${ }^{3}$, \\ Patrick Kuo-Peng ${ }^{3}$, and Laurent Krähenbühl ${ }^{4}$ \\ ${ }^{1}$ University of Liège, Department of Electrical Engineering and Computer Science, ACE, B-4000 Liège, Belgium \\ ${ }^{2}$ F.R.S.-FNRS, Fonds de la Recherche Scientifique, Bruxelles, Belgium \\ ${ }^{3}$ GRUCAD/EEL/UFSC, 88040-970 Florianópolis, Santa Catarina, Brazil \\ ${ }^{4}$ Université de Lyon, Ampère (UMR CNRS 5005), École Centrale de Lyon, F-69134 Écully Cedex, France
}

\begin{abstract}
Model refinements of magnetic circuits are performed via a subdomain finite element method based on a perturbation technique. A complete problem is split into subproblems, some of lower dimensions, to allow a progression from 1-D to 3-D models. Its solution is then expressed as the sum of the subproblem solutions supported by different meshes. A convenient and robust correction procedure is proposed allowing independent overlapping meshes for both source and reaction fields, the latter being free of cancellation error in magnetic materials. The procedure simplifies both meshing and solving processes, and quantifies the gain given by each model refinement on both local fields and global quantities.
\end{abstract}

Index Terms-Finite element method (FEM), magnetic circuit, model refinement, subdomain method.

\section{INTRODUCTION}

$\mathbf{T}$ HE perturbation of finite element (FE) solutions provides clear advantages in repetitive analyses and helps improving the solution accuracy [1]-[6]. It allows to benefit from previous computations instead of starting a new complete FE solution for any variation of geometrical or physical data. It also allows different problem-adapted meshes and computational efficiency due to the reduced size of each subproblem.

A subproblem FE method is herein developed for coupling solutions of various dimensions, starting from simplified models [7], based on ideal flux tubes defining 1-D models, that evolve towards 2-D and 3-D accurate models, allowing leakage flux and end effects. It is an extension of the method proposed in [3]-[5], applied to refinements up to 3-D models. With the former method, material and interface condition changes were combined separately. A more convenient and robust correction procedure is proposed here. It combines both types of changes, via volume and surface sources, in single correction steps. This allows independent overlapping meshes for both source and reaction fields, which simplifies the meshing procedure. This also solves the critical problem of cancellation error in magnetic materials.

The developments are performed for the magnetic vector potential FE magnetostatic formulation, paying special attention to the proper discretization of the constraints involved in each subproblem. The method is illustrated and validated on test problems.

\section{Magnetic SubProblems of VARIOUS Dimensions}

\section{A. Series of Coupled Subproblems}

Instead of solving a complete problem, generally with a 3-D model, it is proposed to split it into a sequence of subproblems,

Manuscript received December 23, 2009; accepted February 10, 2010. Current version published July 21, 2010. Corresponding author: P. Dular (e-mail: Patrick.Dular@ulg.ac.be).

Color versions of one or more of the figures in this paper are available online at http://ieeexplore.ieee.org.

Digital Object Identifier 10.1109/TMAG.2010.2044028 some of lower dimensions, i.e. 1-D and 2-D models, and others performing adequate corrections. The complete solution is then to be expressed as the sum of the subproblem solutions. This offers a way to perform model refinements, with a direct access to each correction, usually of useful physical meaning.

Each subproblem is defined in its own domain, generally distinct from the complete one. At the discrete level, this aims to decrease the problem complexity and to allow distinct meshes with suitable refinements. Each subproblem approximates at best its contribution to the complete solution. The domains of the subproblems usually overlap.

\section{B. Canonical Magnetostatic Problem}

A canonical magnetostatic problem $p$ is defined in a domain $\Omega_{p}$, with boundary $\partial \Omega_{p}=\Gamma_{p}=\Gamma_{h, p} \cup \Gamma_{b, p}$ (possibly at infinity). Subscript $p$ refers to the associated problem $p$. The equations, material relations, boundary conditions (BCs) and interface conditions (ICs) of problem $p$ are

$$
\begin{aligned}
\operatorname{curl} \boldsymbol{h}_{p} & =\boldsymbol{j}_{p}, \quad \operatorname{div} \boldsymbol{b}_{p}=0 \\
\boldsymbol{h}_{p} & =\mu_{p}^{-1} \boldsymbol{b}_{p}+\boldsymbol{h}_{s, p}, \quad \boldsymbol{j}_{p}=\boldsymbol{j}_{s, p}, \\
\boldsymbol{n} \times\left.\boldsymbol{h}_{p}\right|_{\Gamma_{h, p}} & =0,\left.\quad \boldsymbol{n} \cdot \boldsymbol{b}_{p}\right|_{\Gamma_{b, p}}=0 \\
{\left[\boldsymbol{n} \times \boldsymbol{h}_{p}\right]_{\gamma_{p}} } & =\boldsymbol{j}_{f, p}, \quad\left[\boldsymbol{n} \cdot \boldsymbol{b}_{p}\right]_{\gamma_{p}}=\boldsymbol{b}_{f, p},
\end{aligned}
$$

where $\boldsymbol{h}_{p}$ is the magnetic field, $\boldsymbol{b}_{p}$ is the magnetic flux density, $\boldsymbol{j}_{p}$ is the electric current density, $\mu_{p}$ is the magnetic permeability and $\boldsymbol{n}$ is the unit normal exterior to $\Omega_{p}$. The notation $[\cdot]_{\gamma}=$ $\left.\cdot\right|_{\gamma^{+}}-\left.\cdot\right|_{\gamma^{-}}$expresses the discontinuity of a quantity through any interface $\gamma$ (with sides $\gamma^{+}$and $\gamma^{-}$) in $\Omega_{p}$ (the region in between is exterior to $\Omega_{p}$ ).

The fields $\boldsymbol{h}_{s, p}$ and $\boldsymbol{j}_{s, p}$ are volume sources (VSs). The source $\boldsymbol{h}_{s, p}$ is usually used for fixing a remnant induction in magnetic materials. The source $\boldsymbol{j}_{s, p}$ fixes the current density in inductors. With the perturbation method, $\boldsymbol{h}_{s, p}$ is also used for expressing changes of permeability and $j_{s, p}$ for adding portions of inductors [3], [4]. In magnetodynamic problems, $j_{s, p}$ also expresses changes of conductivity [2], [6]. For a change of permeability 
of a region, from $\mu_{q}$ for problem $q$ to $\mu_{p}$ for problem $p$, the VS $\boldsymbol{h}_{s, p}$ in this region is

$$
\boldsymbol{h}_{s, p}=\left(\mu_{p}^{-1}-\mu_{q}^{-1}\right) \boldsymbol{b}_{q}
$$

for the total fields to be related by $\boldsymbol{h}_{q}+\boldsymbol{h}_{p}=\mu_{p}^{-1}\left(\boldsymbol{b}_{q}+\boldsymbol{b}_{p}\right)$.

The surface fields $\boldsymbol{j}_{f, p}$ and $\boldsymbol{b}_{f, p}$ are generally zero, defining classical ICs for the physical fields, i.e. the continuities of the traces $\boldsymbol{n} \times \boldsymbol{h}_{p}$ and $\boldsymbol{n} \cdot \boldsymbol{b}_{p}$. If nonzero, they define possible surface sources (SSs) that account for particular phenomena occurring in the idealized thin region between $\gamma^{+}$and $\gamma^{-}$[2]-[5]. A typical case occurs when, in a problem $q$, the fields $h_{q}$ and $b_{q}$ are explicitly fixed to zero in a region exterior to the studied domain $\Omega_{q}$, separated by the interface $\Gamma_{f, q}$. This amounts to force a discontinuity of the traces $\boldsymbol{n} \times \boldsymbol{h}_{q}$ and/or $\boldsymbol{n} \cdot \boldsymbol{b}_{q}$ through $\Gamma_{f, q}$; usually a BC fixes one of the traces to zero on the inner side $\Gamma_{f, q}^{+}$of $\Gamma_{f, q}$. To restore the missing continuity, a problem $p$ has then to fix the opposite of the discontinuity as an IC through $\Gamma_{f, p}=\Gamma_{f, q}\left(\Gamma_{f, p}\right.$ and $\Gamma_{f, q}$ only differ at the discrete level by their meshes), via

$$
\begin{aligned}
{\left[\boldsymbol{n} \times \boldsymbol{h}_{p}\right]_{\Gamma_{f, p}} } & =\boldsymbol{j}_{f, p}=-\boldsymbol{n} \times\left.\boldsymbol{h}_{q}\right|_{\Gamma_{f, p}^{+}}=-\boldsymbol{n} \times\left.\boldsymbol{h}_{q}\right|_{\Gamma_{f, q}^{+}} \\
{\left[\boldsymbol{n} \cdot \boldsymbol{b}_{p}\right]_{\Gamma_{f, p}} } & =\boldsymbol{b}_{f, p}=-\left.\boldsymbol{n} \cdot \boldsymbol{b}_{p}\right|_{\Gamma_{f, p}^{+}}=-\left.\boldsymbol{n} \cdot \boldsymbol{b}_{p}\right|_{\Gamma_{f, q}^{+}} \cdot
\end{aligned}
$$

Each problem $p$ is to be constrained via the so defined VSs and SSs from parts of the solutions of other problems. This is a key element of the developed method, offering a wide variety of possible corrections, as it will be shown.

\section{Finite ELEMENT WEAK FORMULATIONS}

\section{A. B-Conform Weak Formulations}

The canonical problem $p$ (1a)-(1h) is defined in $\Omega_{p}$ with the magnetic vector potential formulation [3], [4], expressing the magnetic flux density $\boldsymbol{b}_{p}$ in $\Omega_{p}$ as the curl of a magnetic vector potential $\boldsymbol{a}_{p}$. The related $\boldsymbol{a}$-formulation is obtained from the weak form of the Ampère equation (1a), i.e. [3], [4]

$$
\begin{aligned}
& \left(\mu_{p}^{-1} \operatorname{curl} \boldsymbol{a}_{p}, \operatorname{curl} \boldsymbol{a}^{\prime}\right)_{\Omega_{p}}+\left(\boldsymbol{h}_{s, p}, \operatorname{curl} \boldsymbol{a}^{\prime}\right)_{\Omega_{p}}-\left(\boldsymbol{j}_{s, p}, \boldsymbol{a}^{\prime}\right)_{\Omega_{p}} \\
& +\left\langle\boldsymbol{n} \times \boldsymbol{h}_{s, p}, \boldsymbol{a}^{\prime}\right\rangle_{\Gamma_{h, p}}+\left\langle\boldsymbol{n} \times \boldsymbol{h}_{p}, \boldsymbol{a}^{\prime}\right\rangle_{\Gamma_{b, p}} \\
& +\left\langle\left[\boldsymbol{n} \times \boldsymbol{h}_{p}\right]_{\gamma_{p}}, \boldsymbol{a}^{\prime}\right\rangle_{\gamma_{p}}=0, \quad \forall \boldsymbol{a}^{\prime} \in F_{p}^{1}\left(\Omega_{p}\right)
\end{aligned}
$$

where $F_{p}^{1}\left(\Omega_{p}\right)$ is a gauged curl-conform function space defined on $\Omega_{p}$ and containing the basis functions for $\boldsymbol{a}$ as well as for the test function $\boldsymbol{a}^{\prime}$ (at the discrete level, this space is defined by edge FEs); $(\cdot, \cdot)_{\Omega}$ and $\langle\cdot, \cdot\rangle_{\Gamma}$ respectively denote a volume integral in $\Omega$ and a surface integral on $\Gamma$ of the product of their vector field arguments. The surface integral term on $\Gamma_{h, \mathrm{p}}$ accounts for natural BCs of type (1e), usually zero. The term on the surface $\Gamma_{b, p}$ with essential BCs on $\boldsymbol{n} \cdot \boldsymbol{b}_{p}$ is usually omitted because it does not locally contribute to (4). It will be shown to be the key for the post-processing of a solution, a part of which $\boldsymbol{n} \times\left.\boldsymbol{h}_{p}\right|_{\Gamma_{b, p}}$ having to act as a SS.

\section{B. Projections of Solutions Between Meshes}

Some parts of a previous solution $a_{q}$ serve as sources in a subdomain $\Omega_{s, p} \subset \Omega_{p}$ of the current problem $p$. At the discrete level, this means that this source quantity $\boldsymbol{a}_{q}$ has to be expressed in the mesh of problem $p$, while initially given in the mesh of problem $q$. This can be done via a projection method [8] of its curl limited to $\Omega_{s, p}$, i.e.

$$
\begin{aligned}
&\left(\operatorname{curl} \boldsymbol{a}_{q, p-p r o j}, \operatorname{curl} \boldsymbol{a}^{\prime}\right)_{\Omega_{s, p}}=\left(\operatorname{curl} \boldsymbol{a}_{q},\right.\left.\operatorname{curl} \boldsymbol{a}^{\prime}\right)_{\Omega_{s, p}} \\
& \forall \boldsymbol{a}^{\prime} \in F_{p}^{1}\left(\Omega_{s, p}\right)
\end{aligned}
$$

where $F_{p}^{1}\left(\Omega_{s, p}\right)$ is a gauged curl-conform function space for the $p$-projected source $\boldsymbol{a}_{q, p-p r o j}$ (the projection of $\boldsymbol{a}_{q}$ on mesh $p$ ) and the test function $\boldsymbol{a}^{\prime}$. Directly projecting $\boldsymbol{a}_{q}$ (not its curl) would result in significant numerical inaccuracies when evaluating its curl.

\section{VSs for Changes of Material Properties and Inductors}

A change of permeability from problem $q$ to $p$ is taken into account in (4) via the volume integral $\left(\boldsymbol{h}_{s, p}, \operatorname{curl} \boldsymbol{a}^{\prime}\right)_{\Omega_{p}}$. The VS $\boldsymbol{h}_{s, p}$ is given by (2), with $\boldsymbol{b}_{q}=$ curl $\boldsymbol{a}_{q}$. At the discrete level, the source primal quantity $\boldsymbol{a}_{q}$, initially given in mesh $q$, is projected in the mesh $p$ via (5), with $\Omega_{s, p}$ limited to the modified regions. A change of current density in either added or modified inductors is defined in (4) via the source integral $\left(\boldsymbol{j}_{s, p}, \boldsymbol{a}^{\prime}\right)_{\Omega_{p}}$ with the associated $\operatorname{VS} \boldsymbol{j}_{s, p}$.

\section{SSs for Changes of ICs}

With the $\boldsymbol{b}$-conform formulation, ICs (3a) and (3b) are to be defined respectively in weak and strong senses, i.e. via a surface integral and in $F_{p}^{1}\left(\Omega_{p}\right)$. IC (3b) is expressed via a discontinuity of the trace $\boldsymbol{n} \times \boldsymbol{a}_{p}$ through $\Gamma_{f, p}$, i.e.

$$
\left[\boldsymbol{n} \times \boldsymbol{a}_{p}\right]_{\Gamma_{f, p}}=-\boldsymbol{n} \times\left.\boldsymbol{a}_{q}\right|_{\Gamma_{f, q}^{+}} .
$$

As for IC (3a), it is to be weakly expressed via the last integral in (4), with $\gamma_{p}=\Gamma_{f, p}=\Gamma_{f, q}$. The so involved trace $\boldsymbol{- n} \times\left.\boldsymbol{h}_{q}\right|_{\Gamma_{f, q}^{+}}$gains at being kept in a surface integral (weighted by $\boldsymbol{a}^{\prime}$ ), that originally appears in (4) for problem $q$ on $\Gamma_{b, q}$ now restricted to $\Gamma_{f, q}=\Gamma_{f, p}$. It can then be naturally expressed via the other (volume) integrals in (4), i.e.

$$
\begin{aligned}
\left\langle\left[\boldsymbol{n} \times \boldsymbol{h}_{p}\right]_{\Gamma_{f, p}}, \boldsymbol{a}^{\prime}\right\rangle_{\Gamma_{f, p}} & =\left\langle-\boldsymbol{n} \times \boldsymbol{h}_{q}, \boldsymbol{a}^{\prime}\right\rangle_{\Gamma_{f, q}^{+}} \\
& =\left(\mu_{q}^{-1} \operatorname{curl} \boldsymbol{a}_{q}, \operatorname{curl} \boldsymbol{a}^{\prime}\right)_{\Omega_{p}=\Omega_{q}} .
\end{aligned}
$$

At the discrete level, the volume integral in (7) is limited to one single layer of FEs touching $\Gamma_{f, p}^{+}$, because it involves only the associated traces $\boldsymbol{n} \times\left.\boldsymbol{a}^{\prime}\right|_{\Gamma_{f, p}^{+}}$. The source $\boldsymbol{a}_{q}$, initially in mesh $q$, has to be projected in mesh $p$ via (5), with $\Omega_{s, p}$ limited to the FE layer, which thus decreases the computational effort of the projection process.

\section{CONVEnient And Robust Correction Procedure}

Progressions from 1-D to 3-D models have been already studied in [3]-[5], mainly by considering ideal flux tubes with surfaces initially impermeable to the flux, that progressively become permeable to allow leakage flux and end effects. As a drawback of the former approach, the correction problems had to include the inductors together with the magnetic regions, leading thus to classical meshing constraints. Indeed, the correction problems gathered simultaneously the source field and 




Fig. 1. Field lines generated by a stranded inductor: solution of a 1-D model (implicitly extended as constant along the inductor height) $\left(\boldsymbol{b}_{1}\right.$, left $)$, its correction from one coil cap $\left(\boldsymbol{b}_{2}\right.$, middle $)$ and the resulting total field $(\boldsymbol{b}$, right $)$.

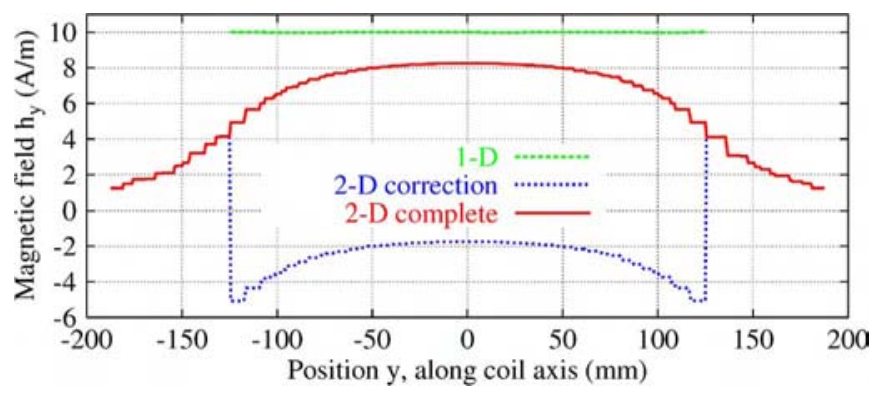

Fig. 2. Magnetic field along the coil axis: $\boldsymbol{h}_{1}$ for the 1-D model, $\boldsymbol{h}_{2}$ for the 2-D correction from the coil cap and $\boldsymbol{h}$ for the complete 2-D model.

leakage flux calculations. The generalized approach developed herein, with both VSs and SSs, enables to make the meshes of the inductors and magnetic regions totally independent, with complete overlapping and with separate calculations of source and leakage fields. Each mesh usually covers unbounded regions via infinite FEs.

\section{A. Step 1: Source Fields Generated by Inductors}

Inductors alone are first studied, with the advantage to benefit from all their symmetries, not broken by additional magnetic regions. A 2-D stranded inductor (Figs. 1 and 2) is first considered as a portion of a infinite solenoid, thus with a 1-D model: $\boldsymbol{b}_{1}$ is constant inside, zero outside and varies linearly in the coil section. This portion is limited by two caps $\Gamma_{f, 1}$ through which the trace $\boldsymbol{n} \cdot \boldsymbol{b}_{1}$ is forced to be discontinuous. The correction problem for end effects compensates this discontinuity via SSs (3b) on the caps. The resulting cap field, calculated in a mesh containing one cap and the surrounding unbounded region, can thus be added to the 1-D solution with adequate translations and rotations towards each coil end, for any coil height. The extension to 3-D coils is straightforward.

Another correction procedure is proposed for 3-D inductors with portions satisfying translational or rotational symmetries, that can be first studied via 2-D models. As an example, a $3-\mathrm{D}$ stranded inductor is defined via the combination of a 2-D plane model for its portion with a translational symmetry and a 2-D axisymmetrical model for its end winding (Figs. 3 and 4). This consists in initially neglecting some end effects, zeroing $\left.\boldsymbol{n} \cdot \boldsymbol{b}_{q}\right|_{\Gamma_{f, q}}$ on the portions caps. Besides, each field is forced to be zero out of each $\Omega_{q}$, which defines a discontinuity of $\boldsymbol{n} \times \boldsymbol{h}_{q}$ through $\Gamma_{f, q}$. With such assumptions, two subproblems 1 and 2 with adjacent non-overlapping sudomains $\Omega_{1}$ and $\Omega_{2}$ share a common interface $\Gamma_{f, 1}=\Gamma_{f, 2}$ through which a combination of field discontinuities occurs. A third subproblem, 3-D, serves



Fig. 3. Field lines generated by a stranded inductor (half geometry): solution of a 2-D plane model in the XY plane $(\mathrm{z}=0)\left(\boldsymbol{b}_{1}\right.$, portion on the left $)$ and of a 2-D axisymmetrical model in the $\mathrm{YZ}$ plane $(\mathrm{x}=0)\left(\boldsymbol{b}_{2}\right.$, portion on the right $)$; the interface between the two portions is shown.

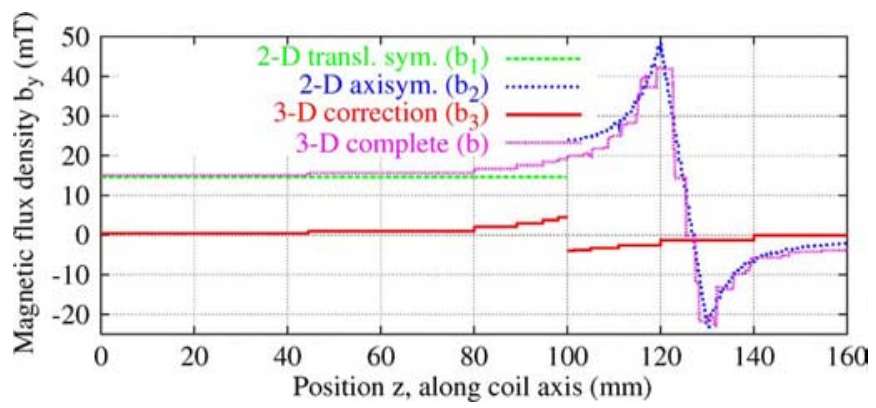

Fig. 4. Magnetic flux density along the coil axis in the 3-D system: $\boldsymbol{b}_{1}$ for the 2-D plane model (implicitly extended as constant up to $\mathrm{z}=100 \mathrm{~mm}$ ), $\boldsymbol{b}_{2}$ for the 2-D axisymmetrical model, $\boldsymbol{b}_{3}$ for the 3 -D correction and $\boldsymbol{b}$ for the complete 3-D model.

then to correct the field distribution in a certain neighborhood $\Omega_{3}$ on both sides of the interface, then denoted $\Gamma_{f, 3}$, via the SSs $\boldsymbol{j}_{f, 3}=-\left(\boldsymbol{n} \times\left.\boldsymbol{h}_{1}\right|_{\Gamma_{f, 1}}-\boldsymbol{n} \times\left.\boldsymbol{h}_{2}\right|_{\Gamma_{f, 2}}\right), \boldsymbol{b}_{f, 3}=0$ (Fig. 4). Because the correction is local to $\Gamma_{f, 3}$, the associated 3-D mesh only needs to be refined in its vicinity. It does not include at all the already considered inductor portions. The solution with such a combination of meshes is generally obtained with a higher accuracy than the solution on a single 3-D mesh, for less computational effort.

\section{B. Step 2: Reaction Fields Free of Cancellation Error}

A first typical problem is that of a magnetic region put in an initially calculated source field $\boldsymbol{b}_{1}$. The associated subproblem 2 is solved in its proper mesh, with the added magnetic core and its infinite surrounding region. The VS (2) is applied, with $\boldsymbol{h}_{s, 2}=\left(\mu_{\text {core }}^{-1}-\mu_{0}^{-1}\right) \boldsymbol{b}_{1}$. The higher $\mu_{\text {core }}$, the lower the total magnetic field $\boldsymbol{h}_{1}+\boldsymbol{h}_{2}$. At the limit, $\boldsymbol{h}_{2}$ approaches $-\boldsymbol{h}_{1}$, which gives an increasing inaccuracy on $\boldsymbol{b}_{2}=\operatorname{curl} \boldsymbol{a}_{2}$ (Fig. 5). A robust way to avoid such a cancellation error is to combine a problem $2 \mathrm{a}$, considering a perfect magnetic core $\left(\mu_{c o r e, 2 a}^{-1}=\right.$ 



Fig. 5. Field lines for the inductor alone $\left(\boldsymbol{b}_{1}\right.$, top left $)$, for the added core $\left(\boldsymbol{b}_{2}\right.$, $\mu_{r, \text { core }}=100$ ) with cancellation error (top right) and robust procedure (bottom right), and for the complete problem $(\boldsymbol{b}$, bottom left $)$; distinct meshes are used for problems 1 and 2 .


Fig. 6. Field lines in the ideal flux tube $\left(\boldsymbol{b}_{1}, \mu_{r, \text { core }}=100\right)$, for the inductor alone $\left(\boldsymbol{b}_{2}\right)$, for the leakage flux $\left(\boldsymbol{b}_{3}\right)$ and for the total field $(\boldsymbol{b})$ (left to right).

0 ), and a problem $2 \mathrm{~b}$, considering a change to the actual permeability $\left(\mu_{\text {core }, 2 b}=\mu_{\text {core }}\right)$. Problem 2a uses a SS $\boldsymbol{j}_{f, 2 \mathrm{a}}=$ $\boldsymbol{- n} \times\left.\boldsymbol{h}_{1}\right|_{\Gamma_{\text {core }}}$, with $\boldsymbol{h}_{2 \mathrm{a}}=0$ and $\boldsymbol{b}_{2 \mathrm{a}} \neq 0$, while problem $2 \mathrm{~b}$ uses the $\operatorname{VS} \hat{h}_{s, 2 \mathrm{~b}}=\left(\mu_{\text {core }}^{-1}-0\right)\left(\boldsymbol{b}_{1}+\boldsymbol{b}_{2 \mathrm{a}}\right)$. Both problems gain at being solved simultaneously, with the SS $\boldsymbol{j}_{f, 2}=\boldsymbol{j}_{f, 2 \mathrm{a}}$ and the resulting relation $h_{2}=\mu_{\text {core }}^{-1} \boldsymbol{b}_{2}+\mu_{\text {core }}^{-1} \boldsymbol{b}_{1}$, with no more inaccuracy (Fig. 5). In practice, this robust procedure asks for the projection of the source field in the added magnetic region as well as in the layer of FEs surrounding this region.

An electromagnet is then studied as an example of low reluctance magnetic circuit [5]. The new procedure for such a circuit splits the problem in a minimum of 3 subproblems (Figs. 6 and 7): (1) the magnetic region and the air gaps considered as an ideal flux tube (with possible start from 1-D models [3]-[5]), (2) the stranded inductor alone, and (3) the consideration of the leakage flux via a SS $\boldsymbol{j}_{f, 3}$ on the flux tube boundary, simultaneously with the change of permeability due to the addition of the magnetic region in the inductor source field (using the extended procedure to avoid the cancellation error). In this way, step 2 and step 3 are based on totally independent meshes; step 1 uses a portion of mesh 3 . All the resulting total solutions have been successfully validated.

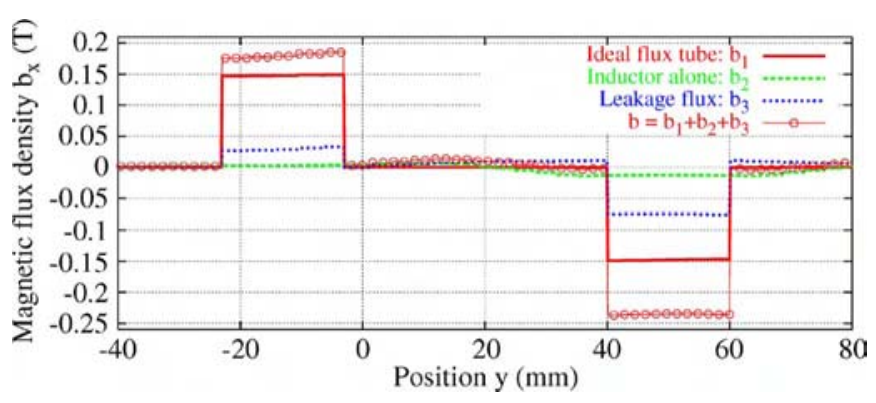

Fig. 7. Magnetic flux density through the horizontal legs of the electromagnet for the ideal flux tube $\left(\boldsymbol{b}_{1}\right)$, for the inductor alone $\left(\boldsymbol{b}_{2}\right)$ and for the leakage flux $\left(\boldsymbol{b}_{3}\right)$; their addition gives the complete solution $(\boldsymbol{b})$.

\section{CONCLusions}

The developed subdomain FE method allows to split magnetic models into subproblems of lower complexity with regard to meshing operations and computational aspects. A natural progression from simple to more elaborate models, from 1-D to 3-D geometries, is thus possible, while quantifying the gain given by each model refinement. From the so calculated field corrections, the associate corrections of global quantities inherent to magnetic models, i.e. fluxes, magnetomotive forces, can be evaluated. Further developments will deal with other model refinements, from static to dynamic, and from linear to nonlinear problems.

\section{ACKNOWLEDGMENT}

This work was supported by the F.R.S.-FNRS (Belgium), the CNPq (Brazil), the Belgian Science Policy (IAP P6/21) and the Walloon Region.

\section{REFERENCES}

[1] Z. Badics, Y. Matsumoto, K. Aoki, F. Nakayasu, M. Uesaka, and K. Miya, "An effective 3-D finite element scheme for computing electromagnetic field distorsions due to defects in eddy-current nondestructive evaluation," IEEE Trans. Magn., vol. 33, no. 2, pp. 1012-1020, 1997.

[2] P. Dular, R. V. Sabariego, J. Gyselinck, and L. Krähenbühl, "Sub-domain finite element method for efficiently considering strong skin and proximity effects," COMPEL, vol. 26, no. 4, pp. 974-985, 2007.

[3] P. Dular, R. V. Sabariego, M. V. Ferreira da Luz, P. Kuo-Peng, and L. Krähenbühl, "Perturbation finite element method for magnetic model refinement of air gaps and leakage fluxes," IEEE Trans. Magn., vol. 45, no. 3, pp. 1400-1403, 2009.

[4] P. Dular, R. V. Sabariego, M. V. Ferreira da Luz, P. Kuo-Peng, and L. Krähenbühl, "Perturbation finite-element method for magnetic circuits," IET Sci., Measure. Technol., vol. 2, no. 6, pp. 440-446, 2008.

[5] P. Dular, R. V. Sabariego, and L. Krähenbühl, "Magnetic model refinement via a perturbation finite element method-From 1-D to 3-D," COMPEL, vol. 28, no. 4, pp. 974-988, 2009.

[6] P. Dular and R. V. Sabariego, "A perturbation method for computing field distortions due to conductive regions with h-conform magnetodynamic finite element formulations,"' IEEE Trans. Magn., vol. 43, no. 4, pp. 1293-1296, 2007.

[7] C. Chillet and J. Y. Voyant, "Design-oriented analytical study of a linear electromagnetic actuator by means of a reluctance network," IEEE Trans. Magn., vol. 37, no. 4, pp. 3004-3011, 2001.

[8] C. Geuzaine, B. Meys, F. Henrotte, P. Dular, and W. Legros, "A Galerkin projection method for mixed finite elements," IEEE Trans. Magn., vol. 35, no. 3, pp. 1438-1441, 1999. 